\title{
Estimating Parameterized Post-Newtonian Parameters from Spacecraft Radiometric Tracking Data
}

\author{
R. S. Park* and D. J. Scheeres ${ }^{\dagger}$ \\ University of Michigan, Ann Arbor, Michigan 48109 \\ G. Giampieri \\ Imperial College, London, England SW7 2BW, United Kingdom \\ and \\ J. M. Longuski ${ }^{\S}$ and E. Fischbach ${ }^{\mathbb{T}}$ \\ Purdue University, West Lafayette, Indiana 47907
}

\begin{abstract}
The theory of general relativity can be tested by precisely measuring small changes in the trajectory of a spacecraft traveling near the sun. An important aspect of such a measurement is the potential of estimating the parameterized post-Newtonian parameters $\gamma$ and $\beta$ independently. We present a detailed covariance analysis of such a trajectory, analyzing uncertainties in the spacecraft state and $\gamma$ and $\beta$. The radiometric data types simulated in our analysis are range, very long baseline interferometry, and Doppler measurements. Also included are the effects of Earth and spacecraft relative geometries, station-location errors, stochastic accelerations, and uncertainties in the solar quadrupole moment $\boldsymbol{J}_{2}$. For an $\mathrm{X}$-band tracking system, we show that if the steady-state stochastic accelerations, station-location, and solar quadrupole moment errors are known better than $10^{-14} \mathrm{~km} / \mathrm{s}^{2}, 0.1 \mathrm{~m}$, and $10^{-8}$, respectively, the experiment can achieve unbiased estimates $\sigma_{\gamma}=8.90 \times 10^{-5}$ and $\sigma_{\beta}=4.09 \times 10^{-4}$. To achieve this level of precision on accelerations requires a drag-free spacecraft or accurate accelerometers.
\end{abstract}

\begin{tabular}{|c|c|}
\hline & Nomenclature \\
\hline $\boldsymbol{A}$ & $=$ linear map of the spacecraft dynamics \\
\hline$a$ & $\begin{aligned} &= \text { total acceleration acting on the } \\
& \text { spacecraft, } \mathrm{km} / \mathrm{s}^{2}\end{aligned}$ \\
\hline $\boldsymbol{a}_{\mathrm{GR}}, \boldsymbol{a}_{J 2}$ & $\begin{aligned}= & \text { acceleration vectors caused by general } \\
& \text { relativity and solar quadrupole moment, } \mathrm{km} / \mathrm{s}^{2}\end{aligned}$ \\
\hline$a_{0}$ & $=$ initial semimajor axis, $\mathrm{km}$ \\
\hline$c$ & $=$ speed of light, $\mathrm{km} / \mathrm{s}$ \\
\hline$E, E_{0}$ & $\begin{aligned}= & \text { actual and initial (Keplerian) sets } \\
& \text { of orbital elements }\end{aligned}$ \\
\hline & $=$ initial eccentricity \\
\hline $\boldsymbol{H}_{R}, \boldsymbol{H}_{V}, \boldsymbol{H}_{D}$ & $\begin{aligned} &= \text { partial derivatives of the range, very long } \\
& \text { baseline interferometry (VLBI), and Doppler } \\
& \text { measurements with respect to the state }\end{aligned}$ \\
\hline $\boldsymbol{I}_{n \times n}$ & $n \times n$ identity matrix \\
\hline$i_{0}$ & initial inclination, deg \\
\hline$J_{2}$ & solar quadrupole moment \\
\hline$k$ & $=$ discrete-time index for measurement times, $\mathrm{s}$ \\
\hline$M_{0}$ & $=$ initial mean anomaly, deg \\
\hline $\boldsymbol{P}$ & $=$ covariance matrix \\
\hline $\boldsymbol{P}_{\omega}$ & covariance caused by process noise \\
\hline$p$ & parameter vector \\
\hline$q$ & measurement model parameter vector \\
\hline
\end{tabular}

Presented as AAS Paper 2003-205 at the AAS/AIAA 2003 Space Flight Mechanics Meeting, Ponce, PR, 9-13 February 2003; received 15 January 2004; revision received 9 June 2004; accepted for publication 10 May 2004. Copyright (C) 2004 by the authors. Published by the American Institute of Aeronautics and Astronautics, Inc., with permission. Copies of this paper may be made for personal or internal use, on condition that the copier pay the $\$ 10.00$ per-copy fee to the Copyright Clearance Center, Inc., 222 Rosewood Drive, Danvers, MA 01923; include the code 0022-4650/05 \$10.00 in correspondence with the CCC.

${ }^{*}$ Graduate Research Assistant, Department of Aerospace Engineering; sanghp@umich.edu. Student Member AIAA.

Associate Professor, Department of Aerospace Engineering; scheeres@ umich.edu. Associate Fellow AIAA.

${ }^{\ddagger}$ Research Associate, Department of Mathematics, The Blackett Laboratory; g.giampieri@ic.ac.uk.

\$Professor, School of Aeronautics and Astronautics; longuski@ ecn.purdue.edu. Associate Fellow AIAA.

"Professor, Physics Department; ephraim@physics.purdue.edu.

\begin{tabular}{|c|c|}
\hline $\boldsymbol{R}$ & $=$ square-root information filter matrix \\
\hline$R_{E}$ & radius of the Earth, $\mathrm{km}$ \\
\hline $\boldsymbol{R}_{H}$ & $\begin{array}{l}=\text { upper triangularized square-root information } \\
\text { filter matrix }\end{array}$ \\
\hline$R_{\odot}$ & $=$ radius of the sun, $\mathrm{km}$ \\
\hline$r$ & norm of the spacecraft position vector, $\mathrm{km}$ \\
\hline $\boldsymbol{r}$ & spacecraft position vector, $\mathrm{km}$ \\
\hline $\boldsymbol{r}_{E}$ & sun-Earth vector, $\mathrm{km}$ \\
\hline$r_{p}$ & spacecraft periapsis radius, $\mathrm{km}$ \\
\hline $\boldsymbol{r}_{\mathrm{sl}}$ & station-location vector, $\mathrm{km}$ \\
\hline $\boldsymbol{r}_{\mathrm{ts}}$ & $\begin{array}{l}=\text { tracking station vector from the } \\
\text { Earth center, } \mathrm{km}\end{array}$ \\
\hline $\boldsymbol{T}_{H}$ & $=$ orthogonal Householder transformation matrix \\
\hline$t, t_{0}$ & current and epoch times, $\mathrm{s}$ \\
\hline$V_{\infty}$ & excess velocity, $\mathrm{km} / \mathrm{s}$ \\
\hline$v$ & norm of the spacecraft velocity vector, $\mathrm{km} / \mathrm{s}$ \\
\hline$v$ & spacecraft velocity vector, $\mathrm{km} / \mathrm{s}$ \\
\hline$v_{p}$ & $=$ spacecraft periapsis velocity, $\mathrm{km} / \mathrm{s}$ \\
\hline$v_{\mathrm{ts}}$ & $\begin{array}{l}=\text { velocity vector from the sun to Earth tracking } \\
\text { station, } \mathrm{km} / \mathrm{s}\end{array}$ \\
\hline$x$ & $=$ spacecraft state vector \\
\hline$y$ & $=$ estimated state vector \\
\hline$Z_{m}, Z_{n}$ & $\begin{array}{l}=\text { longitudinal and latitudinal angular } \\
\text { measurements, deg }\end{array}$ \\
\hline$Z_{R}, Z_{V}, Z_{D}$ & $=$ range, VLBI, and Doppler measurements \\
\hline$\alpha$ & $\begin{array}{l}=\text { right ascension of the Earth tracking } \\
\text { station, deg }\end{array}$ \\
\hline$\beta$ & $\begin{array}{l}=\text { parameterized post-Newtonian parameter } \\
\text { appearing in the } g_{00} \text { component of the } \\
\text { metric tensor }\end{array}$ \\
\hline$\gamma$ & 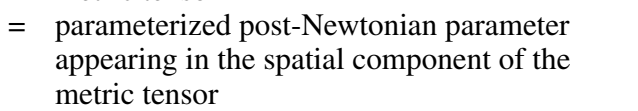 \\
\hline$\Delta V$ & change in spacecraft velocity vector, $\mathrm{km} / \mathrm{s}$ \\
\hline $\bar{\delta}$ & declination of the Earth tracking station, deg \\
\hline$\eta$ & Nordtvedt effect parameter \\
\hline$\Lambda$ & $=$ information matrix \\
\hline$\mu$ & $\begin{aligned}= & \text { gravitational constant of the sun, } \\
& =G M_{\odot}=1.327 \times 10^{11} \mathrm{~km}^{3} / \mathrm{s}^{2}\end{aligned}$ \\
\hline$\xi$ & $\begin{array}{l}=\text { angle of the sun's tangent line from the Earth } \\
\text { center, deg }\end{array}$ \\
\hline
\end{tabular}




\begin{tabular}{|c|c|c|}
\hline$\rho$ & & $\begin{array}{l}\text { range from the Earth tracking station } \\
\text { to the spacecraft, } \mathrm{km}\end{array}$ \\
\hline$\rho$ & $=$ & $\begin{array}{l}\text { position vector of the spacecraft from the Eartl } \\
\text { tracking station, } \mathrm{km}\end{array}$ \\
\hline$\hat{\rho}$ & $=$ & $\begin{array}{l}\text { unit position vector of the spacecraft from the } \\
\text { Earth tracking station }\end{array}$ \\
\hline$\sigma_{a}$ & $=$ & steady-state stochastic accelerations, $\mathrm{km} / \mathrm{s}^{2}$ \\
\hline$\sigma_{i}$ & $=$ & standard deviation of $i$ \\
\hline$\sigma_{i j}$ & $=$ & covariance of $i$ and $j$ \\
\hline$\sigma_{R}, \sigma_{V}, \sigma_{D}$ & $=$ & $\begin{array}{l}\text { approximated range, VLBI, and Doppler } \\
\text { measurement uncertainties }\end{array}$ \\
\hline$\tau$ & $=$ & $\begin{array}{l}\text { correlation time for steady-state stochastic } \\
\text { acceleration, days }\end{array}$ \\
\hline$\Phi$ & $=$ & state transition matrix \\
\hline$\phi$ & $=$ & initial phase angle, deg \\
\hline$\chi$ & $=$ & spacecraft-Earth-sun angle, deg \\
\hline$\psi$ & $=$ & Earth obliquity, deg \\
\hline$\Omega_{0}$ & $=$ & initial argument of ascending node, deg \\
\hline$\omega$ & & argument of perihelion, deg \\
\hline$\omega_{E}$ & $=$ & Earth rotation rate, $\mathrm{deg} / \mathrm{day}$ \\
\hline$\omega_{0}$ & & initial argument of perihelion, deg \\
\hline
\end{tabular}

\section{Introduction}

$\mathbf{T}$ HE theory of general relativity (GR) has been explored with great intensity, both theoretically and experimentally. Previous experimental tests of GR not only improved our understanding of the theory, but also introduced new technologies, such as the Mössbauer effect and atomic clocks in the case of the gravitational red shift. Several GR mission tests, such as Gravity Probe-B, BepiColombo, and the Laser Interferometry Space Antenna, are currently planned to validate and further investigate our modern understanding of gravitational theories.

Tests of GR, such as the precession of Mercury's perihelion and the Viking radar time delay, confirmed the theory by measuring the Eddington-Robertson-Schiff parameters $\gamma$ and $\beta$, which appear in the parameterized post-Newtonian (PPN) formulation. ${ }^{1}$ The parameter $\gamma$ arises in the $g_{i j}$ components of the metric tensor $g_{\mu \nu}$, while $\beta$ characterizes the $g_{00}$ component. Measuring these constants will essentially describe how well GR accounts for the law of gravitation in the weak field limit. In Einstein's formulation of GR, both $\gamma$ and $\beta$ are unity, although both parameters can differ from unity in some alternatives to GR. A recent experiment by Bertotti et al., ${ }^{2}$ in which the radio links with the Cassini spacecraft were calibrated, provided a new estimate $(\gamma-1)=(2.1 \pm 2.3) \times 10^{-5}$. In contrast, the parameter $\beta$ has never been measured independently, but only estimated indirectly from linear combinations involving $\gamma$, such as the perihelion advance effect $(2+2 \gamma-\beta)$, or the Nordtvedt effect, ${ }^{3}$ which measures $\eta=(4 \beta-\gamma-3)$. Let us consider the precession of Mercury's perihelion, where the secular effect of GR on Mercury's trajectory is measured. In this observation, the gravitational shift is highly correlated with the solar quadrupole moment $J_{2}$, a measure of the solar oblateness. If we assume that GR is correct, then our observations can be used to measure the solar quadrupole moment. Alternatively, if we can assume a value for $J_{2}$, an estimate for $\gamma$ and $\beta$ can be found. A recent study by Pireaux and Rozelot ${ }^{3}$ provides an estimated value of the solar quadruople moment $J_{2}=(2.0 \pm 0.4) \times 10^{-7}$. The purpose of the present paper is to verify the feasibility of the test of GR proposed by Longuski et al., ${ }^{4}$ by carrying out a covariance analysis aimed at computing the level of accuracy to which $\gamma, \beta$, and $J_{2}$ can be estimated simultaneously.

A test of GR with use of a heliocentric spacecraft trajectory with small perihelion was previously discussed by Mease et al., ${ }^{5}$ who studied the possibility of estimating the PPN parameters and solar gravitational harmonics by using the proposed Starprobe spacecraft. Their baseline covariance analysis showed potential estimates of the PPN parameter $\gamma$ and solar $J_{2}$ to accuracies of $10^{-2}$ and $10^{-8}$, respectively; however, the parameter $\beta$ was unobservable at the level of $10^{-2}$ because of large station-location uncertainties. In a subsequent study, Mease et al. ${ }^{6}$ carried out a more detailed co- variance analysis including additional quantities of interest, such as the preferred-frame effect parameter $\alpha_{1}$ and the Moffat parameter $\kappa$. However, neither $\gamma$ nor $\beta$ is observable to a significant level using the assumed tracking capability.

More recently, Longuski et al. ${ }^{4}$ have reanalyzed a variation of this test and shown that GR can be tested by precisely measuring small changes in the position of a spacecraft as it escapes from the sun on a hyperbolic trajectory. Their analysis used a heuristic argument, supported by solving the deflection equation for a hyperbolic trajectory, to establish that $\gamma$ and $\beta$ could be measured to an accuracy of $10^{-3}$ assuming foreseeable improvements in spacecraft tracking technology. This theoretical discussion motivated a new consideration of this test of GR. Our follow-on studies based on an analytic approach ${ }^{7}$ verified that the PPN parameters are measurable to the level $\sim 1 \times 10^{-5}$ in principle. Another important result from that study was that simultaneous estimates of the PPN parameters could be obtained separately (i.e., with very little correlation between the estimates of $\gamma$ and $\beta$ ). This result makes the proposed GR experiment unique because other GR tests generally cannot clearly disentangle $\gamma$ and $\beta$ from each other. ${ }^{3}$

The present paper presents a detailed numerical analysis of this test, which includes realistic error sources, such as uncertain nongravitational accelerations, station-location uncertainties, and uncertainty in the solar quadrupole moment, using current and projected measurement accuracies. We first analyze individual contributions from these error sources to the $\gamma$ and $\beta$ estimation accuracies. We then show how these errors degrade the performance of radiometric measurements and discuss the level of precision needed to carry out this experiment at a meaningful level. Also included is the effect of solar occultations, which is a critical problem in the proposed test. This problem is a consequence of the fact that the gravitational shift is at a maximum in proximity of the sun, and a loss of measurements in the early stage of the trajectory significantly degrades the quality of the $\gamma$ and $\beta$ estimates. In addition to the hyperbolic trajectory considered in the previous work, ${ }^{7}$ we also analyze different tracking geometries between the spacecraft and Earth, as well as elliptic orbits with periapsis close to the sun. In practice, an elliptic orbit would be more useful as it can provide multiple passages close to the sun. An elliptic orbit allows for repetition of the experiment, and for longer data arcs near perihelion caused by the slower periapsis speed compared to a hyperbolic orbit. We also present the uncertainty distributions of the PPN parameters as functions of periapsis distance and eccentricity.

Our analysis differs in the following ways from the previous work of Mease et al. ${ }^{6}: 1$ ) very long baseline interferometry (VLBI) measurement is included as a data type in addition to range and Doppler, and 2) more accurate measurement precisions are considered. Furthermore, we ignore PPN parameters other than $\gamma$ and $\beta$ and consider a wider range of spacecraft orbit geometries for the sensitivity study. Over the past few decades, there have been significant improvements in the tracking accuracies of radiometric measurements (e.g., S- to X-band tracking systems). The current implementation of K-band tracking will further enhance our ability to accurately track spacecraft. Future improvement can be anticipated if laser communication is implemented; however, we do not consider this possibility in the present paper. Moreover, VLBI provides highprecision angular measurements of the spacecraft trajectory, a key capability for obtaining better estimates of $\gamma$ and $\beta$ (Ref. 4). In this analysis, we ignore higher-order PPN terms because their effects are negligible. ${ }^{5}$ Also, because each radiometric data type measures a fundamentally different aspect of the GR perturbation, and is sensitive to the relative orbital geometries between the spacecraft and the Earth, we explore a wide range of orbit orientations. Our results exhibit the influence of geometry on the GR estimates as a function of initial phase angle (i.e., the angle $\phi$ shown in Fig. 1). We also include the $J_{2}$ perturbation in the model and study how estimates of this parameter, along with $\gamma$ and $\beta$, vary as a function of inclination, argument of periapsis, and longitude of ascending node. Finally, we show that a mission similar to the proposed Solar Probe mission ${ }^{8}$ can satisfy the trajectory requirements for this test of GR. 


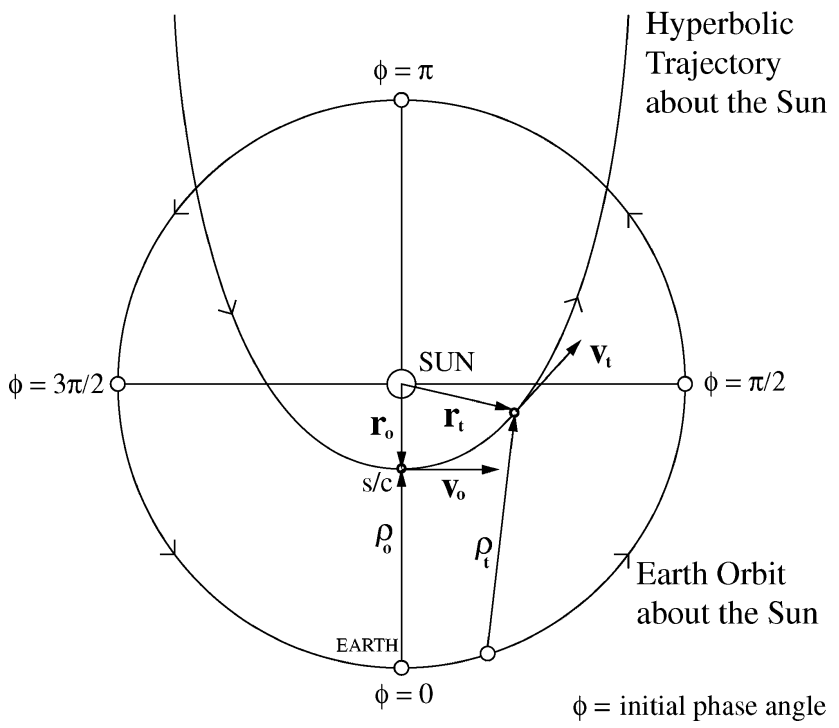

Fig. 1 Hyperbolic flyby of a spacecraft about the sun.

\section{Transient Effects of $\gamma$ and $\beta$}

One can consider the test we are investigating as an extension of previous GR experiments, such as measurements of Mercury's perihelion shift or the deflection of light as a result of the sun's gravity. A novel aspect of this new experiment comes from the potential of separately estimating $\gamma$ and $\beta$. Precisely knowing these predicted parameters allows us to determine how well GR accounts for gravity data. The classic test of GR, observation of the precession of Mercury's perihelion, is based on the secular effect of GR over long time spans. In actuality, the transient oscillations of Mercury's orbit as a result of GR are much larger (over one orbit) than the secular effect; however, their amplitudes are small in absolute terms, and their effect averages out over time. Hence, only the secular effects can be measured over very long time baselines, and these provide estimates of linear combinations of $\gamma$ and $\beta$, such as the perihelion advance effect $(2+2 \gamma-\beta)$ or the Nordtvedt effect $(\eta=4 \beta-\gamma-3)$. Our approach measures transient deviations in the trajectory caused by GR over a short period of time, where the effect is large enough to measure, and this allows us to disentangle $\gamma$ and $\beta$.

If we let $E_{0}$ be a set of Keplerian orbital elements, the change in $E_{0}$ as a result of GR can be defined as $\Delta E=E-E_{0}$, where $E$ is computed by solving the two-body equations of motion incorporating the GR perturbation. The total acceleration acting on the spacecraft is given by

$$
\boldsymbol{a}(t)=-\left(\mu / r^{3}\right) \boldsymbol{r}+\boldsymbol{a}_{\mathrm{GR}}
$$

where $\boldsymbol{a}_{\mathrm{GR}}$ is the GR perturbing acceleration. ${ }^{1}$ Its dimensional representation is

$$
\boldsymbol{a}_{\mathrm{GR}}=\left(\mu / c^{2} r^{3}\right)\left[2(\gamma+\beta)(\mu / r) \boldsymbol{r}-\gamma v^{2} \boldsymbol{r}+2(\gamma+1)(\boldsymbol{r} \cdot \boldsymbol{v}) \boldsymbol{v}\right]
$$

The changes in orbital elements as a result of this perturbation are shown in Fig. 2, where we assume that the spacecraft is initially located at perihelion (four solar radii) with hyperbolic excess velocity $39 \mathrm{~km} / \mathrm{s}$. We note that the GR perturbation acts only in the orbital plane, and therefore the inclination and argument of ascending node are unchanged. The largest change in orbital elements caused by $\gamma$ and $\beta$ occurs very early in the trajectory and essentially disappears after a few days. This is expected because the GR perturbation is at its maximum in close proximity to the sun. One outcome of this observation is a drive to extend radiometric tracking as close to the sun as possible. Furthermore, Longuski et al. ${ }^{7}$ discuss the importance of the partial derivatives of the orbital elements with respect to the GR parameters as a spacecraft travels along its trajectory. An important conclusion from their study is that the partials of the argument of periapsis $\omega$, with respect to $\beta$ and $\gamma$, are unique. The ratios of these partials, which represent the correlation between $\beta$ and $\gamma$,

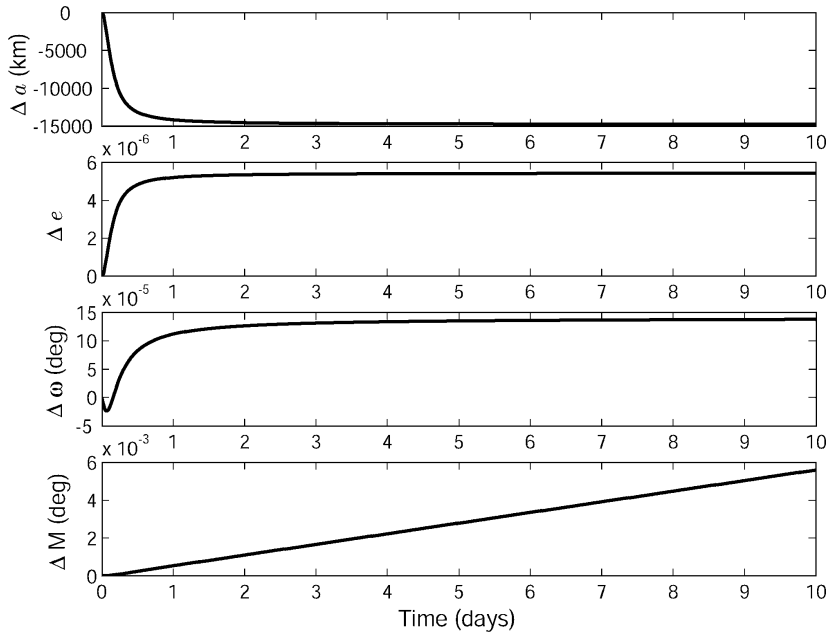

Fig. 2 Change in the semimajor axis $a$, eccentricity $e$, argument of periapsis $\omega$, and mean anomaly $M$ caused by GR.

converge to a constant value slower than the ratios of the partials of the other orbital elements, indicating that there might be sufficient information to disentangle $\gamma$ and $\beta$ by tracking the spacecraft close to perihelion.

In addition to independent $\gamma$ and $\beta$ measurements, our proposed heliocentric spacecraft trajectory offers an opportunity to check GR under a fundamentally different flight regime (i.e., a sub-light-speed experiment). As discussed earlier by Longuski et al., ${ }^{4}$ deflection of a spacecraft trajectory as a result of GR is greater than that of light at the same periapsis distance $r_{p}$. Therefore proper tracking of a spacecraft can, at least in principle, provide PPN estimates that are comparable to the light-speed experiments.

\section{Covariance Analysis}

\section{Baseline State To Be Estimated}

In our numerical approach we follow the baseline trajectory analyzed in Refs. 4 and 7, which places the spacecraft on an escape hyperbolic trajectory at perihelion equal to four solar radii, $r_{p}=4 R_{\odot}$ $\left(R_{\odot}=6.96 \times 10^{5} \mathrm{~km}\right)$. The periapsis velocity is $v_{p}=311 \mathrm{~km} / \mathrm{s}$ with corresponding excess velocity $V_{\infty}=39 \mathrm{~km} / \mathrm{s}$. The nominal trajectory has initial orbital elements of semimajor axis $a_{0}=-0.58$ astronomical unit $(\mathrm{AU})=-8.725 \times 10^{7} \mathrm{~km}$, eccentricity $e_{0}=1.03$, and $i_{0}=\omega_{0}=\Omega_{0}=M_{0}=0 \mathrm{deg}$. The spacecraft and Earth orbits are assumed to be coplanar about a spherical sun. The Earth's orbit is circular with radius of $1 \mathrm{AU}$. The spacecraft trajectory is obtained by numerically integrating two-body equations of motion that incorporate the GR perturbation. While the initial sun-Earth-spacecraft angle (phase angle $\phi$ ) remains as a free parameter, we assume a phase angle of zero for our baseline case.

Our hypothetical trajectory approaches perihelion on an elliptic orbit (with aphelion at Jupiter $\sim 5.2 \mathrm{AU}$ ), then boosts into a hyperbolic escape trajectory (using a perihelion maneuver of $\Delta V \sim 3 \mathrm{~km} / \mathrm{s}$ at $\left.4 R_{\odot}\right)$. Hence, to constrain the initial velocity error we assume the presence of accelerometers to measure the large perihelion burn. At epoch the conservative initial uncertainties (variances) for the initial covariance matrix are $\sigma_{x x}=\sigma_{y y}=\sigma_{z z}=1 \mathrm{~km}^{2}$, $\sigma_{u u}=\sigma_{v v}=\sigma_{w w}=1 \mathrm{~m}^{2} / \mathrm{s}^{2}$, and $\sigma_{\gamma \gamma}=\sigma_{\beta \beta}=1$ with zero correlation. The boost into this hypothetical trajectory introduces new errors. This maneuver is a part of the baseline trajectory only because it was used as a nominal case in earlier studies. We show later that the perihelion boost is not necessarily needed to obtain the same level of $\gamma$ and $\beta$ estimates by analyzing a wider range of trajectories that do not include this maneuver.

In addition to the state and PPN parameters $\gamma$ and $\beta$, we also include other parameters of interest, such as the uncertainties in the station-location vector and in solar $J_{2}$, and study their impact on the $\gamma$ and $\beta$ uncertainties. Our goal is to analyze their individual contributions to the PPN estimates, as this allows us to constrain 
the uncertainties in these parameters and to determine the level of accuracy required in carrying out this experiment. Additionally, we briefly discuss the potential for obtaining improved estimates of these parameters. Lastly, we model the effect of stochastic acceleration perturbations on the trajectory, which can have a critical impact on $\gamma$ and $\beta$ estimation uncertainties.

\section{Definition of the Information and Covariance Matrix}

Our filter model is based on ordinary first-order least-squares approximation theory ${ }^{9}$ and is carried out by using modern orbit determination procedures. ${ }^{10}$ The state vector we estimate in our analysis can be represented as $\boldsymbol{y}(t)=\left[\begin{array}{lll}\boldsymbol{x}^{T} & \boldsymbol{p}^{T} & \boldsymbol{q}^{T}\end{array}\right]^{T}$ for an arbitrary system. For the baseline analysis we define the state vector as $\boldsymbol{y}=\left[\begin{array}{llll}\boldsymbol{r}^{T} & \boldsymbol{v}^{T} & \gamma & \beta\end{array}\right]^{T}$, which assumes no measurement parameters. When additional parameters are included, we simply add them to the state and carry out the same process.

Numerical precision is often lost in the computation of covariance as a result of numerical integration and matrix inversion. A method for retaining precision is to use the square-root information filter $(\mathrm{SRIF})^{9}$ :

$$
\boldsymbol{\Lambda}\left(t_{0} ; t_{k}, t_{0}\right)=\boldsymbol{R}^{T}\left(t_{0} ; t_{k}, t_{0}\right) \boldsymbol{R}\left(t_{0} ; t_{k}, t_{0}\right)
$$

Here $\Lambda\left(t_{0} ; t_{0}, t_{k}\right)$ is the initial state information matrix, which represents the information content of the radiometric data added in the time interval from $t_{0}$ to $t_{k}$, and $\boldsymbol{R}\left(t_{0} ; t_{k}, t_{0}\right)$ is the initial state SRIF matrix, which we propagate, instead of the information matrix, to update data in each time increment. The SRIF matrix is related to the adjoint of the state transition matrix (STM) and ideally maps as

$$
\boldsymbol{R}\left(t_{k} ; t_{k}, t_{0}\right)=\boldsymbol{R}\left(t_{0} ; t_{k}, t_{0}\right) \boldsymbol{\Phi}\left(t_{0}, t_{k}\right)
$$

where $\boldsymbol{\Phi}\left(t_{k}, t_{0}\right)$ describes the linear mapping of the state perturbation about the nominal trajectory. Let $\boldsymbol{T}_{H}$ be an orthogonal Householder transformation matrix ${ }^{9}$ such that the updated SRIF matrix is defined as

$$
\begin{array}{r}
\boldsymbol{R}\left(t_{0} ; t_{k+1}, t_{0}\right)=\boldsymbol{T}_{H}\left[\begin{array}{c}
\boldsymbol{R}\left(t_{0} ; t_{k}, t_{0}\right) \\
\frac{1}{\sigma_{R}} \boldsymbol{H}_{R}\left(t_{k+1}\right) \boldsymbol{\Phi}\left(t_{k+1}, t_{0}\right) \\
\frac{1}{\sigma_{V}} \boldsymbol{H}_{V}\left(t_{k+1}\right) \boldsymbol{\Phi}\left(t_{k+1}, t_{0}\right) \\
\frac{1}{\sigma_{D}} \boldsymbol{H}_{D}\left(t_{k+1}\right) \boldsymbol{\Phi}\left(t_{k+1}, t_{0}\right)
\end{array}\right] \\
=\left[\begin{array}{c}
\boldsymbol{R}_{H}\left(t_{0} ; t_{k+1}, t_{0}\right) \\
0
\end{array}\right]
\end{array}
$$

In the computation, we adopt QR factorization as the Householder transformation. ${ }^{9}$ Here, the $\boldsymbol{H}$ are the partial derivatives of the measurements with respect to the state computed at $t=t_{k+1}$; the subscripts $R, V$, and $D$ denote range, VLBI, or Doppler measurements, respectively. The noise factors (i.e., $\sigma_{R}, \sigma_{V}$, and $\sigma_{D}$ ) are given in Table 1, where they represent the approximate measurement uncertainties provided by S-, X-, and K-band tracking systems. After the Householder transformation is applied, the updated information matrix becomes

$$
\boldsymbol{\Lambda}\left(t_{0} ; t_{k+1}, t_{0}\right)=\boldsymbol{R}_{H}^{T}\left(t_{0} ; t_{k+1}, t_{0}\right) \boldsymbol{R}_{H}\left(t_{0} ; t_{k+1}, t_{0}\right)
$$

and the updated covariance matrix becomes

$$
\begin{aligned}
& \boldsymbol{P}\left(t_{0} ; t_{k+1}, t_{0}\right)=\boldsymbol{\Lambda}^{-1}\left(t_{0} ; t_{k+1}, t_{0}\right) \\
& \quad=\boldsymbol{R}_{H}^{-1}\left(t_{0} ; t_{k+1}, t_{0}\right) \boldsymbol{R}_{H}^{-T}\left(t_{0} ; t_{k+1}, t_{0}\right)
\end{aligned}
$$

Table 1 Summary of measurement accuracies

\begin{tabular}{lccc}
\hline \hline Tracking system & $\sigma_{R}, \mathrm{~m}$ & $\sigma_{V}, \mathrm{nrad}$ & $\sigma_{D}, \mathrm{~mm} / \mathrm{s}^{\mathrm{a}}$ \\
\hline S-band & 10 & 5 & 1 \\
X-band & 1 & 1 & 0.1 \\
K-band & 0.1 & 0.1 & 0.01 \\
\hline \hline
\end{tabular}

${ }^{a}$ For 1-min count time. which represents the evolution of the a priori uncertainties in the state vector.

\section{Computation of the State Transition Matrix}

The STM represents the linear mapping of the state perturbation as a function of time and is defined by the initial spacecraft state vector [i.e., $\boldsymbol{\Phi}\left(t_{k}, t_{0} ; \boldsymbol{r}_{0}, \boldsymbol{v}_{0}, \gamma, \beta\right)$ ]. For the given state vector, the time propagated STM is represented as

$$
\boldsymbol{\Phi}\left(t_{k}, t_{0}\right)=\frac{\partial \boldsymbol{y}(t)}{\partial \boldsymbol{y}\left(t_{0}\right)}
$$

with $\boldsymbol{\Phi}\left(t_{0}, t_{0}\right)$ an $8 \times 8$ identity matrix. The time derivative of the STM is a simple linear relation, $\dot{\boldsymbol{\Phi}}\left(t_{k}, t_{0}\right)=\boldsymbol{A} \boldsymbol{\Phi}\left(t_{k}, t_{0}\right)$, where the linear mapping matrix $\boldsymbol{A}$ is given by

$$
\boldsymbol{A}(t)=\left[\begin{array}{cccc}
0_{3 \times 3} & \boldsymbol{I}_{3 \times 3} & 0_{3 \times 1} & 0_{3 \times 1} \\
\frac{\partial \boldsymbol{a}}{\partial \boldsymbol{r}} & \frac{\partial \boldsymbol{a}}{\partial \boldsymbol{v}} & \frac{\partial \boldsymbol{a}}{\partial \gamma} & \frac{\partial \boldsymbol{a}}{\partial \beta} \\
0_{2 \times 3} & 0_{2 \times 3} & 0_{2 \times 1} & 0_{2 \times 1}
\end{array}\right]
$$

The partial derivatives of the acceleration vector with respect to the state variables yield

$$
\begin{gathered}
\frac{\partial \boldsymbol{a}}{\partial \boldsymbol{r}}=-\frac{\mu}{r^{3}}\left[\boldsymbol{I}_{3 \times 3}-3 \hat{\boldsymbol{r}} \hat{\boldsymbol{r}}^{T}\right]+\frac{\partial}{\partial \boldsymbol{r}}\left(\boldsymbol{a}_{\mathrm{GR}}\right) \sim-\frac{\mu}{r^{3}}\left[\boldsymbol{I}_{3 \times 3}-3 \hat{\boldsymbol{r}} \hat{\boldsymbol{r}}^{T}\right] \\
\frac{\partial \boldsymbol{a}}{\partial \boldsymbol{v}}=\frac{\partial}{\partial \boldsymbol{v}}\left(\boldsymbol{a}_{\mathrm{GR}}\right) \sim 0_{3 \times 3} \\
\frac{\partial \boldsymbol{a}}{\partial \gamma}=\frac{\mu}{c^{2} r^{3}}\left[2 \mu \hat{\boldsymbol{r}}-v^{2} \boldsymbol{r}+2(\boldsymbol{r} \cdot \boldsymbol{v}) \boldsymbol{v}\right] \\
\frac{\partial \boldsymbol{a}}{\partial \beta}=\frac{2 \mu^{2}}{c^{2} r^{3}} \hat{\boldsymbol{r}}
\end{gathered}
$$

where the unit vector $\hat{\boldsymbol{r}}$ is the normalized spacecraft-position vector. The partials of $\boldsymbol{a}_{\mathrm{GR}}$ with respect to spacecraft states (i.e., $\boldsymbol{r}$ and $\boldsymbol{v}$ ) are included in the simulation; however, their contribution is negligible when compared to Newtonian gravity.

\section{Implementing Stochastic Acceleration Effects}

Time-correlated random accelerations can be included in the SRIF matrix as discussed in Scheeres et al. ${ }^{11}$ The perturbations caused by process noise directly feed into the SRIF matrix at time $t_{k}$, and therefore, we solve instead for the SRIF matrix directly rather than solving for the STM. We define the current-state information matrix and related quantities as

$$
\begin{gathered}
\boldsymbol{\Lambda}\left(t_{k} ; t_{k}, t_{0}\right)=\boldsymbol{R}^{T}\left(t_{k} ; t_{k}, t_{0}\right) \boldsymbol{R}\left(t_{k} ; t_{k}, t_{0}\right) \\
\boldsymbol{P}\left(t_{k} ; t_{k}, t_{0}\right)=\boldsymbol{\Lambda}^{-1}\left(t_{k} ; t_{k}, t_{0}\right) \\
\dot{\boldsymbol{R}}\left(t_{k} ; t_{k}, t_{0}\right)=-\boldsymbol{R}\left(t_{k} ; t_{k}, t_{0}\right) \boldsymbol{A}\left(t_{k}\right)
\end{gathered}
$$

The current state-covariance matrix [Eq. (15)] represents the uncertainties in the state variables at time $t_{k}$, whereas the preceding definition is for the a priori estimates. The resulting $\gamma$ and $\beta$ uncertainties do not change because they are not dynamical variables. In the absence of stochastic perturbations, the two definitions of covariance matrices can be mapped into each other by

$$
\boldsymbol{P}\left(t_{k} ; t_{k}, t_{0}\right)=\boldsymbol{\Phi}\left(t_{k}, t_{0}\right) \boldsymbol{P}\left(t_{0} ; t_{k}, t_{0}\right) \boldsymbol{\Phi}^{T}\left(t_{k}, t_{0}\right)
$$

However, this ideal relation is no longer preserved in the presence of stochastic perturbations. The time derivative of the perturbed SRIF matrix is ${ }^{11}$

$$
\begin{aligned}
& \dot{\boldsymbol{R}}\left(t_{k} ; t_{k}, t_{0}\right)=-\boldsymbol{R}\left(t_{k} ; t_{k}, t_{0}\right) \boldsymbol{A}\left(t_{k}\right) \\
& \quad-\frac{1}{2} \boldsymbol{R}\left(t_{k} ; t_{k}, t_{0}\right) \boldsymbol{B} \boldsymbol{P}_{\omega} \boldsymbol{B}^{T} \boldsymbol{R}^{T}\left(t_{k} ; t_{k}, t_{0}\right) \boldsymbol{R}\left(t_{k} ; t_{k}, t_{0}\right)
\end{aligned}
$$


where

$$
\begin{gathered}
\boldsymbol{B}=\left[\begin{array}{ccc}
0_{3 \times 3} & \boldsymbol{I}_{3 \times 3} & 0_{3 \times 2}
\end{array}\right]^{T} \\
\boldsymbol{P}_{\omega}=2 \tau \sigma_{a}^{2} \boldsymbol{I}_{3 \times 3}
\end{gathered}
$$

We assume that the stochastic accelerations are not being estimated during the orbit-determination process and continuously affect the state. For this reason, we map the covariance caused by the process noise $\boldsymbol{P}_{\omega}$ only into the velocity space by using the matrix $\boldsymbol{B}$. In the actual computation the correlation time $\tau$ is set to 0.5 days, and we vary the steady-state acceleration noise to study its impact on $\gamma$ and $\beta$ estimates. Between these measurements, the SRIF matrix is propagated by solving the preceding differential equations, and each measurement is incorporated into the SRIF matrix by

$\boldsymbol{R}\left(t_{k+1} ; t_{k+1}, t_{0}\right)=\boldsymbol{T}_{H}\left[\begin{array}{c}\boldsymbol{R}\left(t_{k+1} ; t_{k}, t_{0}\right) \\ \frac{1}{\sigma_{R}} \boldsymbol{H}_{R}\left(t_{k+1}\right) \\ \frac{1}{\sigma_{V}} \boldsymbol{H}_{V}\left(t_{k+1}\right) \\ \frac{1}{\sigma_{D}} \boldsymbol{H}_{D}\left(t_{k+1}\right)\end{array}\right]=\left[\begin{array}{c}\boldsymbol{R}_{H}\left(t_{k+1} ; t_{k+1}, t_{0}\right) \\ 0\end{array}\right]$

The current-state covariance matrix is then obtained by computing

$$
\boldsymbol{P}\left(t_{k+1} ; t_{k+1}, t_{0}\right)=\boldsymbol{R}_{H}^{-1}\left(t_{k+1} ; t_{k+1}, t_{0}\right) \boldsymbol{R}_{H}^{-T}\left(t_{k+1} ; t_{k+1}, t_{0}\right)
$$

which represents the a posteriori estimates of the state vector at $t=t_{k+1}$.

\section{Extracting $\gamma$ and $\boldsymbol{\beta}$ Estimate Uncertainties}

Each component in a covariance matrix $\sigma_{i j}$ represents the variances of the state or the covariances between two state variables. Hence, we can obtain the uncertainty estimates (i.e., standard deviations) of $\gamma$ and $\beta$ by computing $\sigma_{\gamma}=\sqrt{ } \sigma_{\gamma \gamma}$ and $\sigma_{\beta}=\sqrt{ } \sigma_{\beta \beta}$, respectively. The unique opportunity of the proposed test is the potential of separately estimating $\gamma$ and $\beta$; hence, it is of interest to analyze how these two parameters are correlated. We thus also find the correlation between $\gamma$ and $\beta$ by computing $\left[\sigma_{\gamma \beta} / \sqrt{ }\left(\sigma_{\gamma \gamma} \sigma_{\beta \beta}\right)\right]=\left(\sigma_{\gamma \beta} / \sigma_{\gamma} \sigma_{\beta}\right)$, which can range between -1 and 1 . A value of zero represents a purely uncorrelated measurement, whereas values close to 1 or -1 represent highly correlated or anticorrelated measurements, respectively.

\section{Measurement Data Types and Measurement Partials} with Respect to the State Vector

As mentioned earlier, three different measurement data types are considered. The first data type is two-way radar range measurements,

$$
Z_{R}=\left|\boldsymbol{r}-\boldsymbol{r}_{E}-\boldsymbol{r}_{\mathrm{ts}}\right|=|\rho|=\rho
$$

which measure the distance between the spacecraft and the tracking station based on the travel time of the uplink and downlink signals. Here, $\boldsymbol{r}_{E}$ is the vector from the sun to Earth center, and $\boldsymbol{r}_{\mathrm{ts}}$ is the vector representing the location of the Earth tracking station (Goldstone in our example) relative to the origin at the Earth center. Its analytic representation is given by

$$
\boldsymbol{r}_{\mathrm{ts}}(t)=\left[\begin{array}{ccc}
1 & 0 & 0 \\
0 & \cos \psi & -\sin \psi \\
0 & \sin \psi & \cos \psi
\end{array}\right]\left[\begin{array}{c}
R_{E} \cos \left(\alpha+\omega_{E} t\right) \sin \delta \\
R_{E} \sin \left(\alpha+\omega_{E} t\right) \sin \delta \\
R_{E} \cos \delta
\end{array}\right]
$$

where $\psi$ is the Earth obliquity ( $23.45 \mathrm{deg}$ ), $R_{E}$ is Earth mean radius $(6378 \mathrm{~km}), \alpha$ is the right ascension $(243.17 \mathrm{deg}), \delta$ is the declination (54.67 deg), and $\omega_{E}$ is the Earth inertial rotation rate. We assume that the Earth is initially located along the vernal equinox. We take the partial of $Z_{R}$ with respect to $\boldsymbol{y}$ to find

$$
\boldsymbol{H}_{R}=\frac{\partial Z_{R}}{\partial \boldsymbol{y}}=\left[\begin{array}{lll}
\frac{\partial Z_{R}}{\partial \boldsymbol{r}} & \frac{\partial Z_{R}}{\partial \boldsymbol{v}} & \frac{\partial Z_{R}}{\partial(\gamma, \beta)}
\end{array}\right]=\left[\begin{array}{ll}
\hat{\boldsymbol{\rho}}^{T} & 0_{1 \times 5}
\end{array}\right]
$$

where $\hat{\boldsymbol{\rho}}$ is the unit position vector of the spacecraft from the Earth tracking station.

The second data type we consider is VLBI measurements $Z_{V}$. VLBI measures the longitudinal and latitudinal angles of the spacecraft trajectory in the plane of sky relative to the tracking station. ${ }^{12}$ Combined with range measurements, the three-dimensional position of the spacecraft can be obtained. We represent this measurement as a set of angles

$$
Z_{V}=\left[\begin{array}{ll}
Z_{m} & Z_{n}
\end{array}\right]^{T}
$$

Taking partials with respect to $\boldsymbol{y}$ yields

$$
\boldsymbol{H}_{V}=\frac{\partial Z_{V}}{\partial \boldsymbol{y}}=\left[\begin{array}{cc}
\frac{\hat{\boldsymbol{m}}_{0}^{T}}{\rho} & 0_{1 \times 5} \\
\frac{\hat{\boldsymbol{n}}_{0}^{T}}{\rho} & 0_{1 \times 5}
\end{array}\right]
$$

where we define

$$
\begin{gathered}
\hat{\boldsymbol{l}}_{0}=\hat{\boldsymbol{\rho}} \\
\hat{\boldsymbol{m}}_{0}=\hat{\boldsymbol{l}}_{0} \times \hat{\boldsymbol{n}}_{0} \\
\hat{\boldsymbol{n}}_{0}=\frac{\hat{\boldsymbol{z}}-\left(\hat{z} \cdot \hat{\boldsymbol{l}}_{0}\right) \hat{\boldsymbol{l}}_{0}}{\left|\hat{z}-\left(\hat{z} \cdot \hat{\boldsymbol{l}}_{0}\right) \hat{\boldsymbol{l}}_{0}\right|}
\end{gathered}
$$

and where $\hat{z}=\left[\begin{array}{lll}0 & 0 & 1\end{array}\right]^{T}$

The final data type we consider is Doppler measurements $Z_{D}$,

$$
Z_{D}=\frac{\mathrm{d}}{\mathrm{d} t}\left|\boldsymbol{r}-\boldsymbol{r}_{E}-\boldsymbol{r}_{\mathrm{ts}}\right|=\hat{\boldsymbol{\rho}} \cdot \dot{\boldsymbol{\rho}}
$$

which are widely used for interplanetary missions. This data type gives range rate via Doppler frequency shifts in the transmitted signals and, because of the Hamilton-Melbourne effect, ${ }^{13}$ provide angular information on the trajectory as well. The partial derivative of $Z_{D}$ results in

$$
\boldsymbol{H}_{D}=\frac{\partial Z_{D}}{\partial \boldsymbol{y}}=\left[\begin{array}{lll}
\dot{\boldsymbol{\rho}}^{T}\left(\frac{\partial \hat{\boldsymbol{\rho}}}{\partial \boldsymbol{r}}\right)^{T} & \hat{\boldsymbol{\rho}}^{T} & 0_{1 \times 2}
\end{array}\right]
$$

where

$$
\frac{\partial \hat{\boldsymbol{\rho}}}{\partial \boldsymbol{r}}=\frac{1}{\rho}\left[\boldsymbol{I}_{3 \times 3}-\hat{\boldsymbol{\rho}} \hat{\boldsymbol{\rho}}^{T}\right]
$$

\section{Solar Occultation Effects}

When the spacecraft passes in front of (or behind) the sun (Fig. 3), we cannot obtain radiometric measurements. Because the trajectory originates close to the sun, this can be an important effect in the early stages of the experiment.

Let us define

$$
\chi=\cos ^{-1}\left[\frac{\boldsymbol{\rho} \cdot\left(-\boldsymbol{r}_{E}\right)}{\rho r}\right]
$$

Based on the geometry of the Earth and sun, the angle between $\boldsymbol{r}_{E}$ and the tangent vector from center of the Earth to the outer radius of the sun $\xi$ is computed to be $0.267 \mathrm{deg}$. We assume that no measurements are taken (i.e., $H=0$ ) if $\chi \leq \xi+0.5 \mathrm{deg}$ for Doppler and VLBI measurements and $\chi \leq \xi+5 \mathrm{deg}$ for range measurements. ${ }^{14}$ 


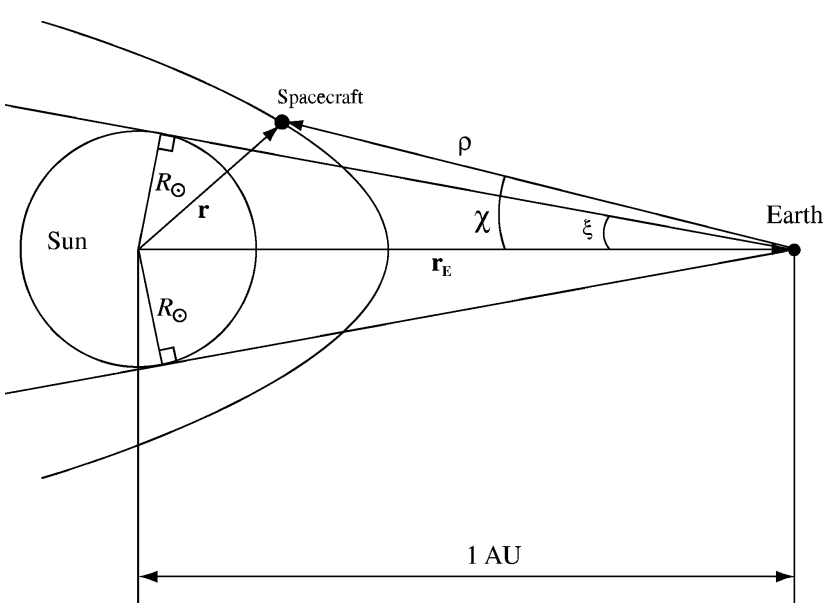

Fig. 3 Occultation effect caused by the sun.

\section{Station-Location Errors}

We define the station-location vector $\boldsymbol{r}_{\mathrm{sl}}$ as

$$
\boldsymbol{r}_{\mathrm{sl}}=\left[\begin{array}{c}
R_{E} \sin \delta \\
R_{E} \cos \delta \\
\alpha
\end{array}\right]
$$

To analyze the effect of uncertainties in $\boldsymbol{r}_{\mathrm{sl}}$ on $\gamma$ and $\beta$ estimates, we include $\boldsymbol{r}_{\mathrm{sl}}$ in the state vector $\boldsymbol{y}$ and carry out the same covariance analysis. The only information needed are the partials of the observation vectors with respect to $\boldsymbol{r}_{\mathrm{sl}}$. Taking partials of range measurements with respect to the station-location vector results in

$$
\frac{\partial Z_{R}}{\partial \boldsymbol{r}_{\mathrm{sl}}}=-\hat{\boldsymbol{\rho}}^{T}\left(\frac{\partial \boldsymbol{r}_{\mathrm{ts}}}{\partial \boldsymbol{r}_{\mathrm{sl}}}\right)
$$

Taking partials of the VLBI measurements with respect to the station-location vector yields

$$
\left[\begin{array}{c}
\frac{\partial Z_{m}}{\partial \boldsymbol{r}_{\mathrm{sl}}} \\
\frac{\partial Z_{n}}{\partial \boldsymbol{r}_{\mathrm{sl}}}
\end{array}\right]=\left[\begin{array}{c}
-\frac{\hat{\boldsymbol{m}}_{0}^{T}}{\rho}\left(\frac{\partial \boldsymbol{r}_{\mathrm{ts}}}{\partial \boldsymbol{r}_{\mathrm{sl}}}\right) \\
-\frac{\hat{\boldsymbol{n}}_{0}^{T}}{\rho}\left(\frac{\partial \boldsymbol{r}_{\mathrm{ts}}}{\partial \boldsymbol{r}_{\mathrm{sl}}}\right)
\end{array}\right]
$$

Finally, the partial derivatives of Doppler measurements are

$$
\frac{\partial Z_{D}}{\partial \boldsymbol{r}_{\mathrm{sl}}}=-\frac{\dot{\boldsymbol{\rho}}^{T}}{\rho}\left(\boldsymbol{I}_{3 \times 3}-\hat{\boldsymbol{\rho}} \hat{\boldsymbol{\rho}}^{T}\right)\left(\frac{\partial \boldsymbol{r}_{\mathrm{ts}}}{\partial \boldsymbol{r}_{\mathrm{sl}}}\right)-\hat{\boldsymbol{\rho}}\left(\frac{\partial \boldsymbol{v}_{\mathrm{ts}}}{\partial \boldsymbol{r}_{\mathrm{sl}}}\right)
$$

where $\boldsymbol{v}_{\mathrm{ts}}$ is the time derivative of $\boldsymbol{r}_{\mathrm{ts}}$.

Uncertainties in station locations corrupt the quality of radiometric measurements and hence can significantly degrade the accuracy of our estimates of $\gamma$ and $\beta$. To analyze this effect, we assume that the uncertainty in the station-location vector is constant and do not estimate $\boldsymbol{r}_{\mathrm{sl}}$ in the covariance computation.

\section{Effect of Solar Quadrupole Moment $\boldsymbol{J}_{2}$}

As discussed by Mease et al., ${ }^{5,6}$ this test also offers the potential of estimating the solar quadrupole moment $J_{2}$ to a precision of order $1 \times 10^{-8}$ for an $\mathrm{X}$-band mission and an order of magnitude better if K-band tracking is employed. Because of large correlations between the PPN parameters and $J_{2}$, we must search the parameter space $\left(\gamma, \beta, J_{2}\right)$ to study their simultaneous contributions to the gravitational shift. ${ }^{3}$ Hence, we include the solar $J_{2}$ as a state variable and estimate it along with $\gamma$ and $\beta$.

To incorporate the solar quadrupole moment in the analysis, we modify the spacecraft acceleration vector by incorporating the perturbing acceleration caused by solar oblateness [i.e.,

$$
\begin{aligned}
& \left.\boldsymbol{a}=-\left(\mu / r^{3}\right) \boldsymbol{r}+\boldsymbol{a}_{\mathrm{GR}}+\boldsymbol{a}_{J 2}\right], \text { where } \\
& \qquad \boldsymbol{a}_{J 2}=\frac{\partial}{\partial \boldsymbol{r}}\left\{\frac{\mu}{r}\left[1+\frac{R_{\odot}^{2} J_{2}}{2 r^{4}}\left(r^{2}-3 z^{2}\right)\right]\right\}
\end{aligned}
$$

Inclusion of $J_{2}$ changes the spacecraft orbit, in particular the orientation of the orbital plane. We analyze orbit orientation $(i, \omega, \Omega)$ to determine the effect of $J_{2}$ on the accuracy of our $\gamma$ and $\beta$ estimates. In this geometric analysis, we only consider a 90-deg inclination case to take maximum advantage ${ }^{6}$ of measuring $J_{2}$, whose nominal value is assumed to be $2 \times 10^{-7}$ as discussed in Ref. 3 . The partial derivatives of the acceleration vector with respect to the spacecraft state are almost negligible, which is expected because the $J_{2}$ acceleration $\boldsymbol{a}_{J 2}$ is even smaller than the GR contribution. The partials with respect to $J_{2}$ are given as

$$
\frac{\partial \boldsymbol{a}}{\partial J_{2}}=-\frac{3 \mu R_{\odot}^{2}}{2 r^{5}}\left[\begin{array}{l}
\left(1-5 \frac{z^{2}}{r^{2}}\right) x \\
\left(1-5 \frac{z^{2}}{r^{2}}\right) y \\
\left(3-5 \frac{z^{2}}{r^{2}}\right) z
\end{array}\right]
$$

\section{Results}

\section{Unbiased Results}

For our analysis, the trajectory conditions given in Refs. 4 and 7 are first verified as the unbiased case, where we assume no error sources are included. The spacecraft is initially located at periapsis of a heliocentric hyperbolic trajectory with $r_{p}=4 R_{\odot}$ and $V_{\infty}=39 \mathrm{~km} / \mathrm{s}$. Figure 4 shows the evolution of PPN uncertainties as a function of time considering S-, X-, and K-band tracking capabilities. An important result is that measurements in the early stage of the trajectory significantly improve the estimates of $\gamma$ and $\beta$. However, as shown in the correlation plot (Fig. 5), early stage measurements have higher correlation factors, and we need to further investigate ways to calibrate radiometric measurements and disentangle the information content for $\gamma$ and $\beta$ estimates. The covariances are linearly proportional to the measurement noise in the absence of stochastic accelerations. Hence, changing measurement accuracies from $\mathrm{X}$ - to K-band tracking can improve $\gamma$ and $\beta$ estimates by an order of magnitude.

The results of the $\gamma$ and $\beta$ estimates, with different time spans, are presented in Table 2 . Taking measurements before periapsis passage significantly reduces the uncertainties in $\gamma$ and $\beta$. This is expected because taking measurements near perihelion provides more useful information about the trajectory. However, for our hyperbolic trajectory, the orbital maneuver at perihelion can introduce problems. Hence we also consider the possibility of obtaining comparable precision with elliptic orbits. All of the values $\sigma_{\gamma}$ and $\sigma_{\beta}$ shown in

Table 2 Summary of unbiased analysis with $X$-band tracking system ${ }^{\mathrm{a}}$

\begin{tabular}{lccc}
\hline \hline $\begin{array}{l}\text { Initial time, } \\
\text { days }\end{array}$ & $\begin{array}{c}\text { Final time, } \\
\text { days }\end{array}$ & $\begin{array}{c}\text { A posteriori } \\
\sigma_{\gamma} \times 10^{4}\end{array}$ & $\begin{array}{c}\text { A posteriori } \\
\sigma_{\beta} \times 10^{4}\end{array}$ \\
\hline $0^{\mathrm{c}}$ & $10^{\mathrm{c}}$ & $0.890^{\mathrm{c}}$ & $4.09^{\mathrm{c}}$ \\
0 & 30 & 0.782 & 3.69 \\
0 & 100 & 0.495 & 3.19 \\
0 & 200 & 0.317 & 0.86 \\
-5 & 5 & 0.607 & 1.12 \\
-10 & 10 & 0.581 & 1.08 \\
-15 & 15 & 0.526 & 0.95 \\
-100 & 100 & 0.123 & 0.24 \\
\hline
\end{tabular}

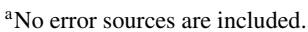

${ }^{\mathrm{b}} \mathrm{With}$ respect to perihelion passage.

${ }^{\mathrm{c}}$ Unbiased case. 

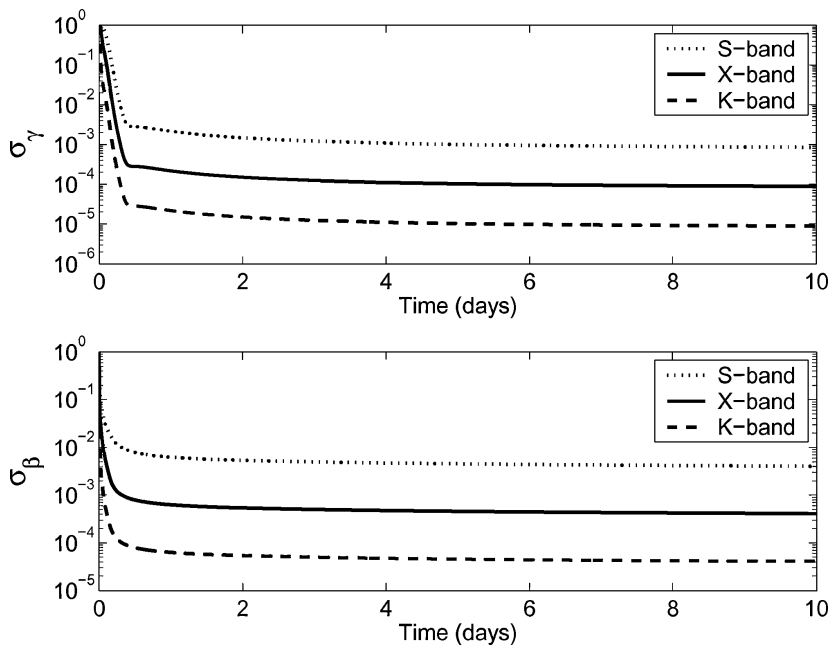

Fig. 4 Uncertainties of $\gamma$ and $\beta$ as functions of time, excluding error sources.

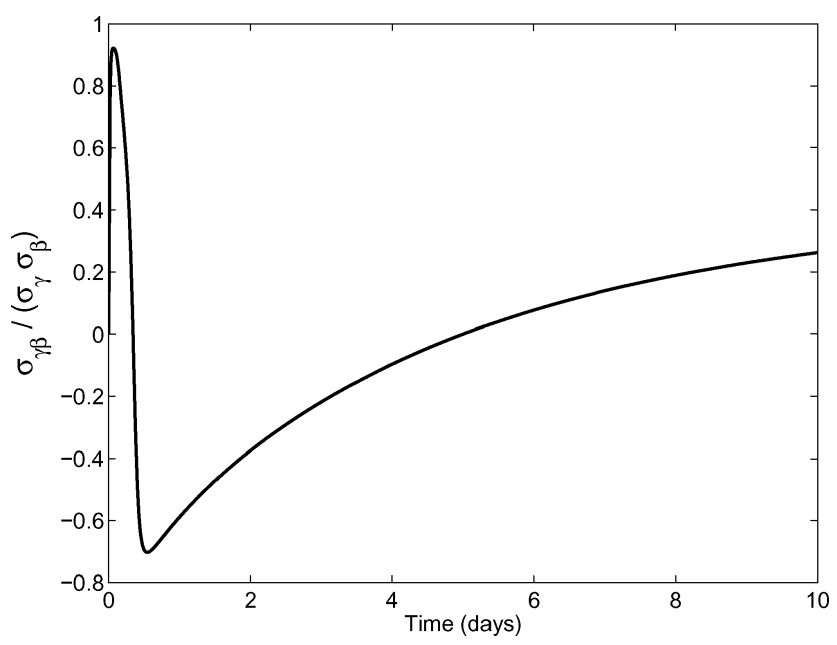

Fig. 5 Correlation between $\gamma$ and $\beta$ estimates as a function of time with $\mathrm{X}$-band tracking accuracies, excluding error sources.

Table 2 are the final ones (a posteriori) taken at the end of the timespan. These uncertainties will be decreased by an order of magnitude when K-band tracking systems are implemented, implying that both $\gamma$ and $\beta$ can be estimated with accuracies better than $10^{-5}$, at least in principle. The parameter $\gamma$ can be estimated more accurately than $\beta$, which is the result obtained by Mease et al. ${ }^{5,6}$ as well.

Figure 6 shows the uncertainties and correlations between $\gamma$ and $\beta$ with different initial phase angles, with and without the solar occultation effect. The estimates are taken at the end of a 10-day timespan, where the spacecraft starts from its perihelion. The uncertainties are relatively sensitive to the initial phase angle $\phi$. This plot shows that there is an order-of-magnitude difference in the estimates when different spacecraft-Earth geometries are considered, thus emphasizing the importance of trajectory design for this experiment. The inclusion of the VLBI measurements as an additional data type did not contribute a significant improvement in $\gamma$ and $\beta$ estimates when compared to Mease et al.; hence, the accurate range and Doppler measurements and finding the optimal spacecraft orientation are the keys in obtaining improved estimates of the PPN parameters.

For the remainder of this paper, by "unbiased case" we mean tracking the spacecraft for 10 days from perihelion by using X-band measurement noise (i.e., the first line of Table 2). We used this result as a standard as it represents the true information content our measurements can extract from the trajectory.
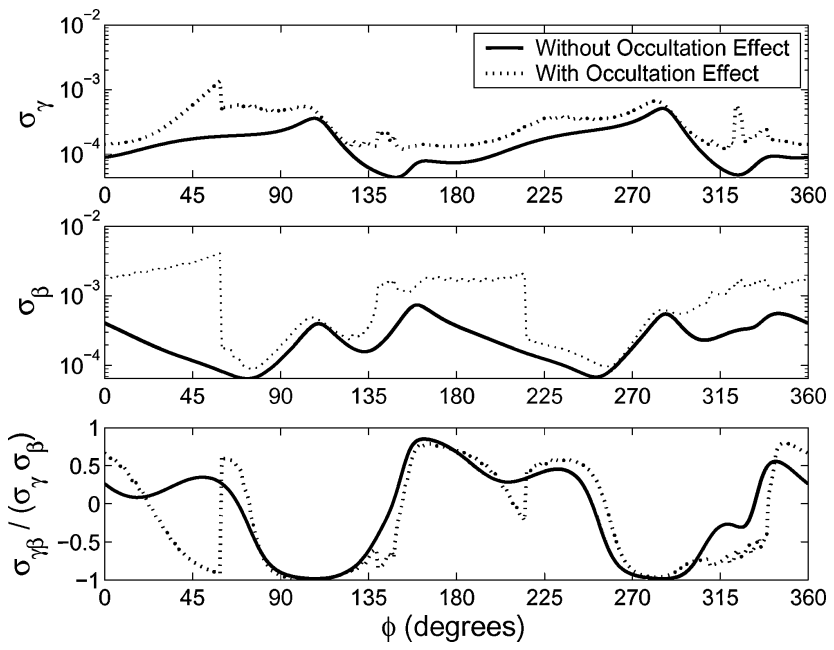

Fig. 6 Uncertainty and correlation of $\gamma$ and $\beta$ estimates as functions of initial sun-Earth-spacecraft angle, excluding error sources.
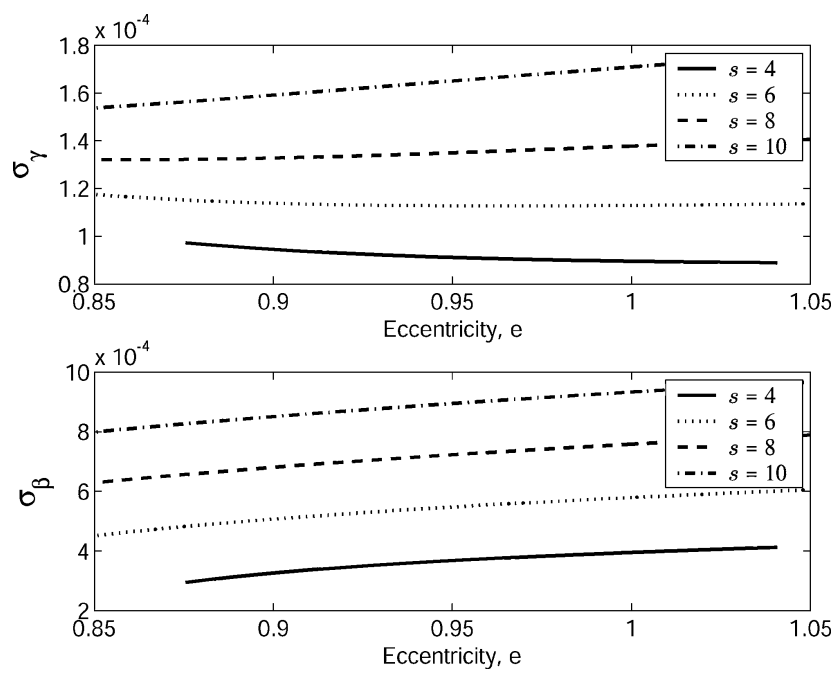

Fig. 7 Accuracies of $\gamma$ and $\beta$ estimates as functions of eccentricity $\boldsymbol{e}$, excluding the error sources $\left(s=r_{p} / R_{\odot}\right)$.

\section{Elliptic Orbits}

As mentioned earlier, the longer data arcs and repeated measurements possible with an elliptic orbit might improve our estimation results. Figure 7 shows the sensitivity of uncertainties in $\gamma$ and $\beta$ as a function of eccentricity for various periapses, where $e<1$ represents an elliptic orbit. This figure shows that the overall change in the uncertainty distribution for a fixed $r_{p}$ is not significant, which means that there is a possibility of conducting this test using elliptic orbits. Also restricting the periapsis radius to $r_{p}=4 R_{\odot}$ is not necessary: $r_{p}$ can be increased to lower the heat shield requirement and thus mission cost. However, when the solar $J_{2}$ is included, it might still be desirable to minimize periapsis distance because the $J_{2}$ uncertainty is highly sensitive to $r_{p}$ (Ref. 5). For orbits with $r_{p}=4 R_{\odot}$, the estimate of $\beta$ becomes more accurate as eccentricity decreases, whereas the estimates of $\gamma$ are better with hyperbolic orbits. Considering the repetition in tracking measurements, increased data arcs in the vicinity of the sun, and absence of a large maneuver at periapsis, elliptic orbits provide an opportunity for more accurate estimation of $\gamma$ and $\beta$. This assumes that the spacecraft is capable of enduring multiple close passes of the sun.

\section{Effect of Stochastic Accelerations}

The effect of stochastic accelerations on the state variables (i.e., position and velocity components) is usually negligible over a short period of time; however, their effect on $\gamma$ and $\beta$ uncertainties is 
Table 3 Effect of stochastic acceleration ${ }^{a}$

\begin{tabular}{lcc}
\hline \hline $\begin{array}{l}\text { Steady-state acceleration } \\
\sigma_{a}, \mathrm{~km} / \mathrm{s}^{2}\end{array}$ & $\begin{array}{c}\text { A posteriori } \\
\sigma_{\gamma} \times 10^{4}\end{array}$ & $\begin{array}{c}\text { A posteriori } \\
\sigma_{\beta} \times 10^{4}\end{array}$ \\
\hline $10^{-10}$ & 465 & 231 \\
$10^{-11}$ & 63.3 & 42.8 \\
$10^{-12}$ & 7.53 & 8.62 \\
$10^{-13}$ & 1.57 & 5.18 \\
$10^{-14}$ & 0.971 & 4.41 \\
Unbiased $^{\mathrm{b}}$ & 0.890 & 4.09 \\
\hline \hline
\end{tabular}

${ }^{\mathrm{a}}$ Covariances are evaluated at the end of a 10-day time span. ${ }^{\mathrm{b}}$ Assumes no stochastic acceleration.
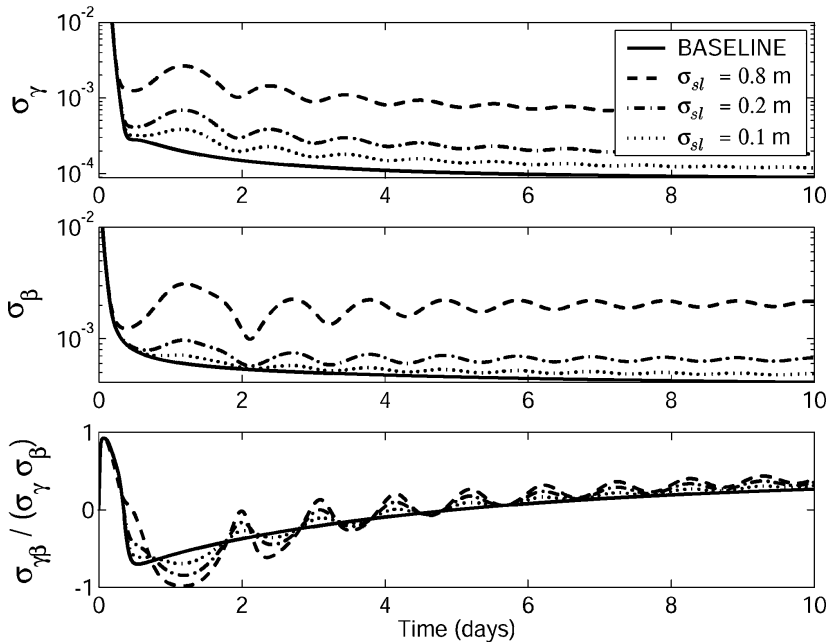

Fig. 8 Effect of station-location errors on the estimates of $\gamma$ and $\beta$ with $\mathrm{X}$-band tracking noises.

significant. Table 3 shows how the process noise affects overall tracking performance. As seen from the table, the $10^{-3}$ precision for $\gamma$ and $\beta$ discussed in Ref. 4 is obtainable when $\sigma_{a}=10^{-12} \mathrm{~km} / \mathrm{s}^{2}$. If the steady-state uncertainty is known to better than $10^{-14} \mathrm{~km} / \mathrm{s}^{2}$, we can achieve the unbiased estimates; however, this will require either accurate onboard accelerometers or a drag-free spacecraft.

\section{Effect of Station-Location Errors}

Figure 8 presents the time responses of $\sigma_{\gamma}$ and $\sigma_{\beta}$ when stationlocation errors of $0.8,0.2$, and $0.1 \mathrm{~m}$ are considered. As seen, stationlocation uncertainties $\sim 0.1 \mathrm{~m}$ will ensure that our desired accuracy will be achieved. Fortunately, current technology provides this level of precision, and hence X-band radiometric measurements can provide useful information about the GR contribution to the trajectory. However, reducing the noise level by use of K-band tracking does not necessarily improve the $\gamma$ and $\beta$ uncertainties because simultaneous increases in station-location precision are required. Therefore, if the $\mathrm{K}$-band tracking system is implemented, it might be more practical to estimate the station-location vector along with $\gamma$ and $\beta$. Also shown in the plot are oscillations in $\sigma_{\gamma}$ and $\sigma_{\beta}$. If the station location is estimated, $\sigma_{\gamma}$ and $\sigma_{\beta}$ will always decrease without oscillation.

\section{Effects of Solar Quadrupole Moment}

Table 4 shows $\sigma_{\gamma}$ and $\sigma_{\beta}$ when $J_{2}$ is included for a spacecraft orbit with zero inclination. Considering the $J_{2}$ uncertainty as an unestimated perturbation causes a dramatic degradation in the $\gamma$ and $\beta$ estimates as a result of the high correlations between $\beta$ and $J_{2}$. Hence, we include $J_{2}$ in our state estimates. Table 4 shows that the estimate of $\beta$ is highly sensitive to initial uncertainties in $J_{2}$, which explains why $\beta$ has not been estimated independently from the precession of Mercury's perihelion (i.e., either $J_{2}$ or $\beta$ must be known accurately to measure the other).

To understand the effect of orbit orientation, we show the uncertainty distributions of $\gamma, \beta$, and $J_{2}$ as functions of $\omega$ and $\Omega$ in Figs. 9-11. We consider a $90-\mathrm{deg}$ inclination with a priori
Table 4 Effect of solar quadrupole moment ${ }^{\mathrm{a}}$

\begin{tabular}{lccc}
\hline \hline A priori & $\begin{array}{c}\text { A posteriori } \\
\sigma_{\gamma} \times 10^{4}\end{array}$ & $\begin{array}{c}\text { A posteriori } \\
\sigma_{\beta} \times 10^{4}\end{array}$ & $\begin{array}{c}\text { A posteriori } \\
\sigma_{J_{2}} \times 10^{8}\end{array}$ \\
\hline $10^{-5}$ & 0.894 & 27.8 & 3.15 \\
$10^{-6}$ & 0.894 & 27.7 & 3.15 \\
$10^{-7}$ & 0.893 & 26.5 & 3.00 \\
$10^{-8}$ & 0.890 & 9.26 & 0.953 \\
$10^{-9}$ & 0.890 & 4.18 & 0.1 \\
Unbiased & 0.890 & 4.09 & $0^{\mathrm{b}}$ \\
\hline \hline
\end{tabular}

${ }^{\mathrm{a}}$ Covariances are evaluated at the end of a 10-day time span. ${ }^{\mathrm{b}}$ Assumes $\sigma_{J 2}=0$.

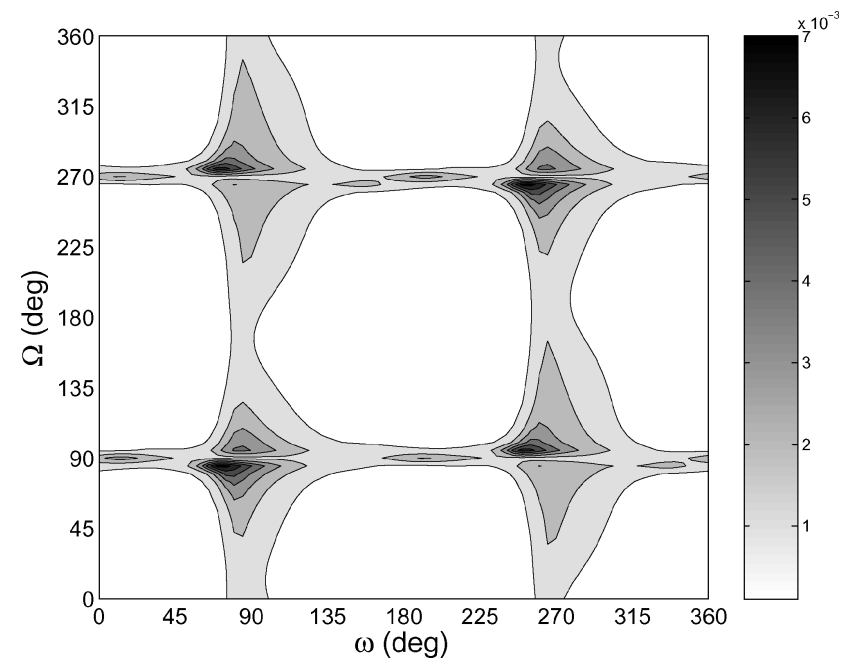

Fig. 9 Uncertainty of $\gamma$ as a function of $\omega$ and $\Omega$ with $X$-band tracking noises and 90-deg inclination.

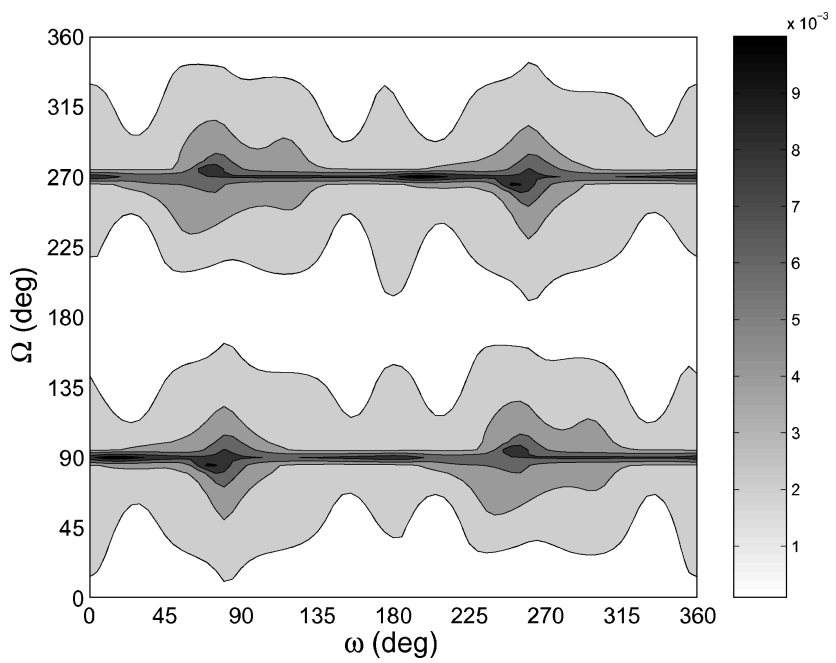

Fig. 10 Uncertainty of $\beta$ as a function of $\omega$ and $\Omega$ with $X$-band tracking noises and 90-deg inclination.

$\sigma_{J_{2}}=10^{-6}$. One important result to note is that $\sigma_{\gamma}, \sigma_{\beta}$, and $\sigma_{J_{2}}$ are highly sensitive to spacecraft orbit orientations, analogous to the sensitivity of $\sigma_{\gamma}$ and $\sigma_{\beta}$ to the initial phase angle. When solar occultation effects are included, the range of uncertainties changes, but the basic trend of the uncertainty distributions remains the same. The estimates become highly degraded near $\Omega= \pm 90 \mathrm{deg}$. One clear explanation comes from the fact that the spacecraft orbit is perpendicular to the Earth line of sight, decreasing the information content of both range and Doppler. 
Table 5 Accumulated effect of error sources ${ }^{\mathrm{a}}$

\begin{tabular}{lcccccc}
\hline \hline & & & $\mathrm{A}$ & $\mathrm{A}$ & $\mathrm{A}$ & $\mathrm{A}$ \\
Data & $\begin{array}{c}\sigma_{a}, \\
\mathrm{~km} / \mathrm{s}^{2}\end{array}$ & $\begin{array}{c}\sigma_{\mathrm{sl}}{ }^{\mathrm{b}} \\
\mathrm{m}\end{array}$ & $\begin{array}{c}\text { priori } \\
\sigma_{J_{2}} \times 10^{8}\end{array}$ & $\begin{array}{c}\text { posteriori } \\
\sigma_{\gamma} \times 10^{4}\end{array}$ & $\begin{array}{c}\text { posteriori } \\
\sigma_{\beta} \times 10^{4}\end{array}$ & $\begin{array}{c}\text { posteriori } \\
\sigma_{J_{2}} \times 10^{8}\end{array}$ \\
\hline X-band & $10^{-12}$ & 0.1 & 1 & 12.6 & 75.3 & 0.999 \\
X-band & $10^{-14}$ & 0.1 & 1 & 1.87 & 24.8 & 0.981 \\
K-band & $10^{-12}$ & 0.1 & 1 & 65.0 & 113 & 1.29 \\
K-band & $10^{-14}$ & 0.1 & 1 & 2.17 & 26.7 & 3.01 \\
K-band & $10^{-14}$ & 0.05 & 1 & 1.10 & 14.1 & 1.58 \\
K-band & $10^{-14}$ & 0.01 & 1 & 0.295 & 6.03 & 0.622 \\
K-band & $10^{-15}$ & 0.01 & 1 & 0.227 & 3.09 & 0.435 \\
K-band & $10^{-16}$ & 0.001 & 0.1 & 0.144 & 1.88 & 0.0971 \\
Unbiased & 0 & 0 & 0 & 0.890 & 4.09 & 0 \\
\hline
\end{tabular}

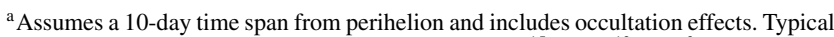
mission uncertainty caused by nongravitational forces ${ }^{15}$ is $10^{-12} \mathrm{~km} / \mathrm{s}^{2}(1-\sigma)$.

${ }^{\mathrm{b}}$ Accuracy of the Earth-tracking station location.

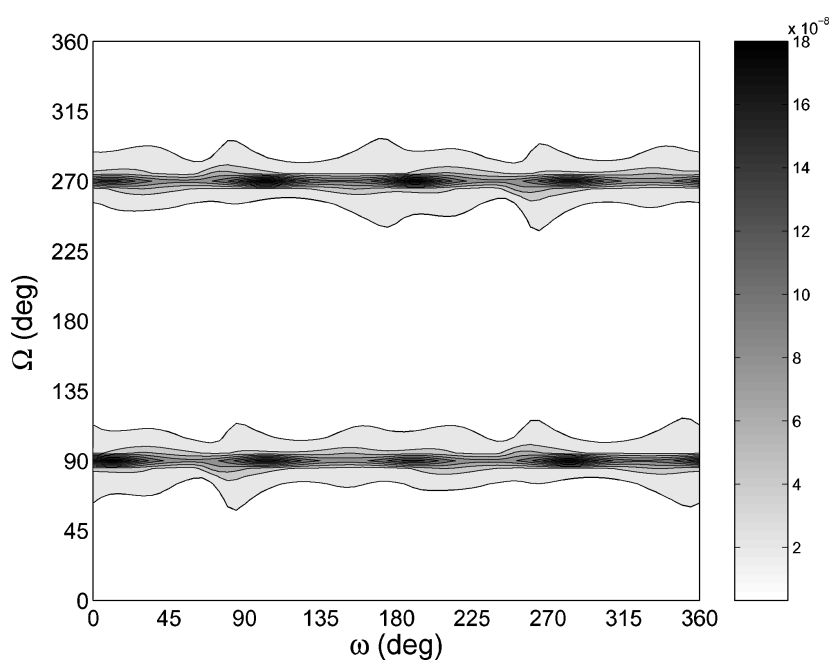

Fig. 11 Uncertainty of $J_{2}$ as a function of $\omega$ and $\Omega$ with $X$-band tracking noises and 90-deg inclination.

\section{Accumulated Effect of Error Sources}

The preceding results have exhibited the effects of individual error sources on estimates of $\gamma$ and $\beta$. We now discuss how accurately $\gamma$ and $\beta$ can be estimated when all of the error sources are accumulated in the estimation filter.

Table 5 shows how the estimates of $\gamma, \beta$, and $J_{2}$ vary when different error uncertainties are included in the filter model. The unbiased accuracies are not obtainable with these assumed error bounds; however, uncertainties with accumulated errors can still be compared to current estimates of $\gamma$ and $\beta$. We also see from this table that a decrease in the measurement noise to the K-band tracking capability requires simultaneous reduction in the relevant error sources (e.g., station-location errors), which is expected. We can conclude from this table that the most challenging task of this experiment is to accurately assess the nongravitational forces acting on the spacecraft near the sun, which, according to Longuski et al., ${ }^{15}$ are typically $10^{-12} \mathrm{~km} / \mathrm{s}^{2}$.

Finally, Table 6 provides $\gamma, \beta$, and $J_{2}$ estimates when different spacecraft orbits are analyzed. For these results we apply more realistic error bounds on the process noise and on $J_{2}$ to show the current feasibility of this test. Again, $\sigma_{\gamma}, \sigma_{\beta}$, and $\sigma_{J_{2}}$ are sensitive to the relative geometries. The last result, where $i=90 \mathrm{deg}, \omega=180 \mathrm{deg}$, and $\Omega=225 \mathrm{deg}$, represents an orbit analogous to the baseline case considered in Mease et al. ${ }^{5,6}$

\section{Comparison of Baseline and Solar Probe Trajectories}

At present, the mission that could feasibly carry out this new test of general relativity is the Solar Probe mission, ${ }^{8}$ which is still at a preapproval stage at NASA. The apoapsis distance of the current mission plan is approximately 5.2 AU (at Jupiter) with the periapsis
Table 6 Effect of spacecraft orientation ${ }^{\mathrm{a}}$

\begin{tabular}{lccccc}
\hline \hline$i$, & $\omega$, & $\Omega$, & A posteriori & A posteriori & A posteriori \\
deg & deg & deg & $\sigma_{\gamma} \times 10^{4}$ & $\sigma_{\beta} \times 10^{4}$ & $\sigma_{J_{2}} \times 10^{8}$ \\
\hline 0 & 0 & 0 & 12.6 & 75.3 & 0.999 \\
45 & 0 & 0 & 12.7 & 75.0 & 0.996 \\
90 & 0 & 0 & 12.7 & 75.2 & 0.987 \\
45 & 45 & 45 & 33.5 & 52.3 & 0.989 \\
90 & 45 & 45 & 8.7 & 58.6 & 0.953 \\
90 & 180 & 225 & 16.6 & 59.2 & 0.970 \\
\hline \hline
\end{tabular}

a Assumes $\sigma_{a}=10^{-12} \mathrm{~km} / \mathrm{s}^{2}$, station-location error $=0.1 \mathrm{~m}, \sigma_{J 2}=10^{-8}$ (a priori), and includes solar occultations.

at $4 R_{\odot}$, which corresponds to an eccentricity of $\sim 0.99$ and a period of approximately four years. Figure 7 indicates that the resulting uncertainties in $\beta$ are favorable with this elliptic orbit. The hyperbolic trajectory (with $e=1.03$ ) provides a slightly better result for $\gamma$ (but not for $\beta$ ); however, Solar Probe's multiple perihelion passages will allow for an overall increase in the level of accuracy. To carry out this experiment, considering the large nongravitational disturbances present near the sun, the spacecraft might need to be equipped with precision accelerometers or a drag-free system. We believe that our results indicate that there is a realistic possibility of carrying out this new GR experiment as an add-on component to the Solar Probe mission objectives.

\section{Conclusions}

In this paper, we have analyzed how well the parameterized post-Newtonian (PPN) parameters $\gamma$ and $\beta$ can be estimated from spacecraft radiometric tracking data, such as range, very long baseline interferometry, and Doppler measurements. In the baseline case, the spacecraft starts from a perihelion at four solar radii with $v_{p}=311 \mathrm{~km} / \mathrm{s}$, and we obtain radiometric measurements as it propagates along the heliocentric trajectory. Various error sources are included in estimates of $\gamma$ and $\beta$, including stochastic acceleration, station-location errors, and solar $J_{2}$. Although this is a preliminary analysis, it captures most of the fundamentals required for evaluation of this experiment. Several crucial characteristics of the uncertainties in $\gamma$ and $\beta$ are obtained from this analysis. The first important conclusion is that placing a spacecraft on a heliocentric elliptic orbit yields results that are essentially equivalent to those obtained from a hyperbolic orbit. Additionally, an elliptic orbit provides multiple perihelion passages, which will enhance the estimation accuracy of $\gamma, \beta$, and $J_{2}$. Analysis shows that it might be feasible to carry out this test as part of the Solar Probe mission, which is currently under development. To do so, some improvements in spacecraft-tracking technology will be required. The effect of stochastic accelerations significantly degrades the accuracy of the experiment, and hence it is necessary to decrease the steady-state uncertainty in nongravitational accelerations by either using accurate accelerometers or by implementing a drag-free spacecraft. Station-location errors degrade the accuracies of the PPN parameters, but current estimates appear to be adequate for X-band tracking. Simultaneous reduction in station-location error is required for a K-band tracking system. Finally, the solar $J_{2}$ can be measured accurately from this experiment.

We have demonstrated that under ideal conditions general relativity can be tested to a significant accuracy by tracking spacecraft trajectories near the sun and have shown that such an experiment is feasible.

\section{Acknowledgments}

The research described in this paper was sponsored by the Interplanetary Network Directorate Technology Program at the Jet Propulsion Laboratory, California Institute of Technology, which is under contract with NASA. The work of E. Fischbach was supported in part by the U.S. Department of Energy under Contract DE-AC02-76ER071428. 


\section{References}

${ }^{1}$ Will, C. M., Theory and Experiment in Gravitational Physics, Cambridge Univ. Press, Cambridge, England, U.K., 1993, Sec. 7.

${ }^{2}$ Bertotti, B., Iess, L., and Tortora, P., "A Test of General Relativity Using Radio Links with the Cassini Spacecraft," Nature, Vol. 425, No. 6956, 2003 , pp. 374-376.

${ }^{3}$ Pireaux, S., and Rozelot, J.-P., "Solar Quadrupole Moment and Purely Relativistic Gravitation Contributions to Mercury's Perihelion Advance," Astrophysics and Space Science, Vol. 284, No. 4, 2003, pp. 1159-1194.

${ }^{4}$ Longuski, J. M., Fischbach, E., and Scheeres, D. J., "Deflection of Spacecraft Trajectories as a New Test of General Relativity," Physical Review Letters, Vol. 86, No. 14, 2001, pp. 2942-2945.

${ }^{5}$ Mease, K. D., Wood, L. J., Bergam, M. J., and White, L. K., "Estimation of Solar Gravitational Harmonics with Starprobe Radiometric Tracking Data," Journal of the Astronautical Sciences, Vol. 31, No. 1, 1983, pp. 3-22.

${ }^{6}$ Mease, K. D., Anderson, J. D., Wood, L. J., and White, L. K., "Tests of General Relativity Using Starprobe Radio Metric Tracking Data," Journal of Guidance, Control, and Dynamics, Vol. 7, No. 1, 1983, pp. 36-44.

${ }^{7}$ Longuski, J. M., Fischbach, E., Scheeres, D. J., Giampieri, G., and Park, R. S., "Deflection of Spacecraft Trajectories as a New Test of General Relativity: Determining the PPN Parameters $\beta$ and $\gamma$," Physical Review D, Vol. 69, 2004, 042001.

${ }^{8}$ Anderson, J. D., Colombo, G., Friedman, L. D., and Lau, E. L., "An Arrow to the Sun," Gravitazione Sperimentale, International Meeting on
Experimental Gravitation, Accademia Nazionale dei Lincei, Rome, 1977, pp. 393-422.

${ }^{9}$ Montenbruck, O., and Gill, E., Satellite Orbits, 2nd ed., Springer-Verlag, Berlin, 2001, pp. 257-291.

${ }^{10}$ Bierman, G. J., Factorization Methods for Discrete Sequential Estimation, Academic Press, New York, 1977.

${ }^{11}$ Scheeres, D. J., Han, D., and Hou, Y., "Influence of Unstable Manifolds on Orbit Uncertainty," Journal of Guidance, Control, and Dynamics, Vol. 24, No. 3, 2001, pp. 573-585.

${ }^{12}$ Thurman, S. W., and Sybert, C. B., "Planetary Approach Orbit Determination Using Earth-Based Short and Long Baseline Radio Interferometry," Advances in the Astronautical Sciences, Vol. 76, Pt. 2, 1991, pp. 1039-1053.

${ }^{13}$ Hamilton, T. W., and Melbourne, W. G., "Information Content of a Single Pass of Doppler Data from a Distant Spacecraft," JPL Space Programs Summary, Vol. 3, May 1966, pp. 18-23.

${ }^{14}$ Morabito, D. D., Shambayati, S., Finley, S., and Fort, D., "The Cassini May 2003 Solar Conjunction," IEEE, Vol. 51, No. 2, 2003, pp. 201-219.

${ }^{15}$ Longuski, J. M., Todd, R. E., and Koenig, W. W., "Survey of Nongravitational Forces and Space Environmental Torques: Applied to the Galileo," Journal of Guidance, Control, and Dynamics, Vol. 15, No. 3, 1992, pp. 545-553.

D. Spencer Associate Editor 